\title{
Perineural invasion in oral cancer: challenges, controversies and clinical impact
}

\author{
Sabrina Daniela da Silva ${ }^{1,2,3}$, Luiz Paulo Kowalski ${ }^{4}$ \\ ${ }^{1}$ Department of Otolaryngology Head and Neck Surgery, ${ }^{2}$ Segal Cancer Centre and Lady Davis Institute for Medical Research, Sir Mortimer B. \\ Davis-Jewish General Hospital, Quebec, Canada; ${ }^{3}$ Departments of Medicine, Oncology, and Pharmacology and Therapeutics, Faculty of Medicine, \\ McGill University, Quebec, Canada; ${ }^{4}$ Department of Head and Neck Surgery and Otorhinolaryngology, A.C. Camargo Cancer Center and National \\ Institute of Science and Technology on Oncogenomics (INCITO), Brazil \\ Correspondence to: Luiz Paulo Kowalski, MD, PhD. Director, Department of Head and Neck Surgery and Otorhinolaryngology, A.C. Camargo \\ Cancer Center, Rua Professor Antonio Prudente, 211, 01509-900 Sao Paulo, Brazil. Email: lp_kowalski@uol.com.br. \\ Comment on: Yang X, Tian X, Wu K, et al. Prognostic impact of perineural invasion in early stage oral tongue squamous cell carcinoma: Results from \\ a prospective randomized trial. Surg Oncol 2018;27:123-8.
}

Submitted Oct 25, 2018. Accepted for publication Nov 15, 2018.

doi: $10.21037 /$ cco.2018.11.04

View this article at: http://dx.doi.org/10.21037/cco.2018.11.04

\section{Introduction}

Oral squamous cell carcinoma (OSCC) corresponds to $95 \%$ of head and neck cancers and it is the $6^{\text {th }}$ most frequent cancer worldwide (1). Although numerous advances in diagnosis and treatment have been reported, the overall 5 -year survival rate for OSCC remains almost unchanged within the past three decades (2). OSCC is characterized by invasive growth patterns, frequently associated with perineural invasion (PNI), high risk of regional lymph node metastases, and a considerable rate of loco-regional recurrences and second primary cancers. Many patients have advanced-stage tumors at diagnosis that must be managed with aggressive multimodality treatment that frequently cause significant morbidity and mortality (3).

OSCC is not a single and uniform disease. In fact, it includes a heterogeneous group of neoplasms that rises from the accumulation of numerous epigenetic and genetic modifications, frequently associated with exposure to tobacco and alcohol carcinogens, resulting in the activation and overexpression of oncogenes and inactivation of tumor suppressors genes. These genetic modifications confer advantages in cell division and survival to the cells, such as growth factor-independent proliferation, resistance to apoptotic signaling, and an enhanced capability to move through the extracellular matrix (ECM) and invade adjacent tissues (4). One of the most difficult barriers to overcome in the treatment of OSCC is the ability of cancer cells to disrupt the basal lamina, degrade ECM, release themselves from the primary lesion, escape immune system, gain access to lymphatic and blood vessels, and establish metastasis at regional lymph nodes and distant sites (5).

Treatment decisions in OSCC are mostly dependent on tumor site, TNM classification, pathologic parameters and patient's clinical status and preferences. Several studies have showed the significance of pathologic findings, such as the type of invasive edge, inflammatory response, stromal component, tissue eosinophilia, and PNI (6). Interactions between epithelial tumor cells and the associated stroma are a powerful connection that may influence cancer initiation and disease progression. The perineural space provides an appropriate tumor microenvironment for the growth of cells from neurotropic malignancies, probably due to the secretion of cellular factors [such as brain derived neurotrophic factor (BDNF), the glial cell line derived neurotrophic factor (GDNF), the neural cell adhesion molecule (NCAM), the nerve growth factor (NGF), substance P (SP), and chemokines] (7) and the presence of the respective receptors that attract cancer cells and stimulate their growth and proliferation along the nerves $(5,8)$. Clinical, histological, and biological features of PNI have been studied extensively in several types of cancer. In head and neck cancers, the tumor spread is often done along the major nerves, which serve as conduits for intracranial extension. Trigeminal and their branches [cranial nerve $(\mathrm{CN}) \mathrm{V}]$, facial $(\mathrm{CN}$ VII) are 
the most commonly involved nerves. They also present an extensive network of fibers and interconnections between them that may serve for tumor cell migration. PNI is often asymptomatic. However, neural spread may cause specific symptoms such as pain, paresthesia, muscle weakness, numbness and formication. Sometimes, in advanced cases, complete denervation may induce muscle atrophy (9). The role of these alterations in the management of patients with OSCC is still controversial and open to debate. It is clear and necessary a better understanding of the biological and molecular mechanisms to target PNI as part of cancer therapy for patients with advanced OSCC.

The majority of head and neck carcinomas are tobacco and alcohol-related, occur in older patients, affect tongue and floor of the mouth and has well-established morphological prognostic factors such as pattern of invasion, tumor grade, and PNI (10). However, in the last three decades, the advent and increasing occurrence of human papillomavirus (HPV) infection resulted in significant changes in the clinical presentation, treatment planning and outcomes. The majority of HPV-related carcinomas are non-keratinizing squamous cell carcinoma that are more responsive to surgery and radiotherapy with a favourable prognosis, opening the opportunity to investigate new treatment protocols aiming treatment de-escalation (11). The specific biological and molecular mechanisms underlying the expression of these histologic phenotypes (keratinizing versus non-keratinizing) are unclear. Interaction between HPV oncoproteins, specifically E6 and E7, modulate cell cycle progression through the regulation of TP53 and RB functions and it affects cell growth and differentiation, cell immortalization, and prevent apoptosis (12). It is likely that these alterations may be responsible for the development specific microscopic alterations and specific types of metastatic spread (such as PNI) that need to be explored.

\section{PNI}

PNI is a distinct form of metastatic spread independent of lymphatic or vascular invasion (8). This route of metastasis may difficult the capability to establish local disease control because tumor cells can trek along nerve tracts and migrate from primary tumor for distant sites that can be missed during surgery procedures (5). Then, patients can present pain due to the persistent growth with a long clinical course. One limitation of the literature relating to PNI is the absence of a clear standardized definition/classification or method for detection (13). Cruveilheir described
PNI in head and neck cancer for the first time in 1835 . Batsakis (1985) gave a more general definition of PNI as tumor cell invasion in, around and through the nerves. In 2009, Liebig et al. (8) proposed the most referenced and accepted PNI description: "tumor in close proximity to nerve and involving at least $33 \%$ of its circumference or tumor cells within any of the 3 layers of the nerve sheath or tumor is identified in close proximity to the nerve and involves more than one-third of its circumference" $(5,8,13)$. However, these definitions do not make a clear differentiation between "perineural spread" or the detection of cancer cells in and around the perineural space, and "intraneural spread" or the penetration of cancer cells inside the nerve; these details might be difficult to identify by histological section but could have significant impact in tumor prognosis $(5,14)$. We prefer to consider PNI as positive on finding tumor cells in the perineural space or epineurium. Some studies have broadly defined PNI as tumor cells touching a nerve $(13,15)$. Another concern is the method for detection to identify PNI in histological analysis considering the biopsy procedure, the laboratory protocol to prepare the slides, and the number of tissue sections examined $(5,13)$. There is a huge variation in the incidence of reported PNI in OSCC, from a low frequency between $2 \%$ to a high of $82 \%$, specifically when neural stains were used (16). This marked variation reflects the absence of a standardized definition/ classification and the lack of a clear method for PNI detection (13). Consequently, this scenario contributes to the inconsistent conclusions reached by different studies regarding the prognostic impact of PNI in OSCC due to subjectivity and errors of underreporting. The value of PNI in OSCC associated with poor prognosis and the lack of target approaches reflect the need of molecular studies involved in communication between cancer and neural cells.

To the best of our knowledge, there are no published studies focusing on specific characteristics of PNI, such as the type, extension, and quality in OSCC, especially as it associates with the clinical impact. In OSCC, only the assessment of presence or absence of invasion in the perineural space is a required component of pathologic analysis according to reporting protocols published by the College of American Pathologists and the Royal College of Pathologists in the United Kingdom (17). PNI is present in both primary and recurrent tumors, irrespective of its histologic grade and it should be checked and reported in every OSCC surgical specimen. Although some studies have concluded that PNI is an independent predictive factor for local and regional recurrence, others did not find 
Table 1 Manuscripts describing the prognostic impact of patients with head and neck squamous cell carcinoma with PNI

\begin{tabular}{|c|c|c|c|c|}
\hline Author (year) & Site & $\begin{array}{l}\text { Tumor } \\
\text { classification }\end{array}$ & $\begin{array}{l}\text { Number of } \\
\text { patients }\end{array}$ & Prognostic impact \\
\hline Fagan et al. (1998) & HNSCC & $\mathrm{T} 1-4$ & 142 & $\begin{array}{l}\mathrm{PNI} \text { increased risk of loco-regional recurrence and is an } \\
\text { independent predictive factor of extracapsular spread }\end{array}$ \\
\hline Liao et al. (2008) & $\mathrm{OC}$ & $\mathrm{T} 1-3$ & 460 & $\begin{array}{l}\text { No significant differences in 5-year local control and } \\
\text { overall survival rates }\end{array}$ \\
\hline Tai et al. (2011) & $\mathrm{OC}$ & $\mathrm{T} 1-2$ & 307 & $\begin{array}{l}\text { Elective neck dissection is indicative for patients with } \\
\text { PNI-positive tumors to improve disease specific survival } \\
\text { and neck control }\end{array}$ \\
\hline Miller et al. (2012) & HNSCC & $\mathrm{T} 1-4$ & 142 & $\begin{array}{l}\text { PNI is correlated with nodal status, T stage and } \\
\text { disease-free survival }\end{array}$ \\
\hline Chen et al. (2013) & $\mathrm{OC}$ & $\mathrm{T} 1-2$ & 442 & $\begin{array}{l}\text { PNI was not significant risk factors for the OSCC neck } \\
\text { control and overall survival }\end{array}$ \\
\hline Lazer et al. (2013) & HNSCC & $\mathrm{T} 1-4$ & 291 & $\begin{array}{l}\text { PNI and lymph node invasion tend toward loco-regional } \\
\text { metastasis }\end{array}$ \\
\hline $\begin{array}{l}\text { Chatzistefanou et al. } \\
\text { (2014) }\end{array}$ & $\mathrm{OC}$ & $\mathrm{T} 1-3$ & 78 & $\mathrm{PNI}$ is an independent predictor for cervical metastasis \\
\hline Shen et al. (2014) & $\mathrm{OC}$ & $\mathrm{T} 1-4$ & 116 & $\begin{array}{l}\text { PNI can predict the progression of tongue cancer and } \\
\text { prognosis of OSCC patients }\end{array}$ \\
\hline Jardim et al. (2015) & $\mathrm{OC}$ & $\mathrm{T} 1-4$ & 142 & $\begin{array}{l}\text { PNI has impact on survival outcomes in patients with } \\
\text { advanced OSCC tumors }\end{array}$ \\
\hline Laske et al. (2016) & $\mathrm{OC}$ & $\mathrm{T} 1-4$ & 72 & $\begin{array}{l}\text { PNI in early disease has a particularly high impact on } \\
\text { survival }\end{array}$ \\
\hline Joo et al. (2016) & Hypopharyngeal & $\mathrm{T} 1-4$ & 105 & $\begin{array}{l}\text { PNI was an independent predictor factor for cervical } \\
\text { lymph nodes }\end{array}$ \\
\hline Anand et al. (2017) & $\mathrm{OC}$ & $\mathrm{T} 1-4$ & 83 & $\begin{array}{l}\text { PNI indicative of worse prognosis despite adjuvant } \\
\text { treatment }\end{array}$ \\
\hline Kim et al. (2018) & $\mathrm{OC}$ & $\mathrm{T} 1-4$ & 373 & $\begin{array}{l}\text { PNI was correlated with tumor site and lymph nodes } \\
\text { metastasis }\end{array}$ \\
\hline Nair et al. (2018) & $\mathrm{OC}$ & $\mathrm{T} 1-4$ & 152 & $\begin{array}{l}\text { PNI worsens survival and warrants intensification of } \\
\text { adjuvant therapy }\end{array}$ \\
\hline Cracchiolo et al. (2018) & $\mathrm{OC}$ & $\mathrm{T} 1-4$ & 382 & PNI predicts worse disease-free-survival \\
\hline Baumeister et al. (2018) & $\mathrm{OC}$ & $\mathrm{T} 1-4$ & 178 & PNI was marker for aggressive tumor growth \\
\hline Yang et al. (2018) & $\mathrm{OC}$ & $\mathrm{T} 1-2$ & 221 & $\begin{array}{l}\text { PNI predict cervical metastasis, loco-regional relapse } \\
\text { and poor survival outcome }\end{array}$ \\
\hline
\end{tabular}

OC, oral cancer; OSCC, oral squamous cell carcinoma; HNSCC, head and neck squamous cell carcinoma; PNI, perineural invasion. Table inspired on the manuscript published by Bur et al. 2016 (13). 
any significative association and its role as an independent prognosticator for local relapse and cervical metastasis failed in these studies (Table 1) $(15,17-20)$. Another issue is that many publications have a relatively low number of cases and included different subsites and tumor stages, which might explain the wide discrepancies. The majority of the investigations have shown that PNI is associated with OSCC loco-regional recurrence, distant metastasis and decreased 5-year overall survival probability (Table 1). Although these findings support PNI as an adverse risk feature, it remains unclear how PNI should be exactly measured, whether PNI should be quantified rather than only be reported as presence versus absence, and how the identification of PNI should affect the clinical management of OSCC patients (21). The presence of PNI has also been shown to mark late stage disease and one of the factors used to decide about adjuvant radiotherapy/radiochemotherapy (including the subgroup of node-negative patients) (6). Some studies even suggest the need for elective neck dissection in PNI-positive node negative tumors and radical surgery associated with adjuvant treatment for the advanced stage tumors (5). Recently, Yang et al. (2018) in a prospective randomized trial from China showed that elective neck dissection did not reduce the rate of locoregional relapse and/or improve disease specific survival in early-stage OSCC patients with PNI (17). However, these authors also suggested that further investigation is necessary considering a larger cohort of PNI early-stage OSCC patient population to clarify and then guide the optimal procedures for neck management.

Recently, the National Comprehensive Cancer Network panel (NCCN) published updates to the NCCN Clinical Practice Guidelines in Oncology (NCCN Guidelines ${ }^{\circledR}$ ) and NCCN Drugs \& Biologics Compendium (NCCN Compendium $^{\circledR}$ ) for head and neck cancers indicating radiotherapy as an adjuvant therapy option for patients with perineural/vascular/lymphatic invasion following surgical resection of a T1-T2, N0 lesion (22). Although intensitymodulated radiotherapy (IMRT) has reduced toxicity compared to standard conformal radiotherapy techniques, xerostomia, oral infections, dental caries, pain, taste loss, and difficulty speaking and swallowing remain the critical sequelae with significant impact in the quality of life for OSCC patients. It is the responsibility of the clinicians to discuss thoroughly with patients the potential benefits and toxicity associated with adjuvant therapy to allow them to make an informed decision concerning the treatment.

\section{Conclusions}

PNI is a challenge to the multidisciplinary head and neck cancer management. The presence of PNI necessitates more aggressive treatment. The excision surgery must involve the nerve path until clear margins are achieved. The management of neck lymph nodes and the adjuvant therapy is mandatory; the intensity-modulated radiation therapy offer advantages to reduce toxicity and side effects and therefore improve quality of life for patients with OSCC. Understanding the mechanism of tumor progression and the molecular events that promote PNI in OSCC can pave way to the development of therapeutic agents that target this form of tumor spread.

\section{Acknowledgments}

None.

\section{Footnote}

Conflicts of Interest: The authors have no conflicts of interest to declare.

\section{References}

1. Cancer Research. Available online: https://www. cancerresearchuk.org. Accessed September 2018.

2. Kowalski LP, Magrin J, Sabóia M, et al. Squamous cell carcinoma of the tongue: a review of 629 patients treated at a single institution. South Am J Cancer 1997;1:245-54.

3. da Silva SD, Ferlito A, Takes RP, et al. Advances and applications of oral cancer basic research. Oral Oncol 2011;47:783-91.

4. Hanahan D, Weinberg RA. Hallmarks of cancer: the next generation. Cell 2011;144:646-74.

5. Binmadi NO, Basile JR. Perineural invasion in oral squamous cell carcinoma: a discussion of significance and review of the literature. Oral Oncol 2011;47:1005-10.

6. Varsha BK, Radhika MB, Makarla S, et al. Perineural invasion in oral squamous cell carcinoma: Case series and review of literature. J Oral Maxillofac Pathol 2015;19:335-41.

7. Chilton JK. Molecular mechanisms of axon guidance. Dev Biol 2006;292:13-24.

8. Liebig C, Ayala G, Wilks JA, et al. Perineural invasion in cancer: a review of the literature. Cancer 2009;115:3379-91.

9. Frunza A, Slavescu D, Lascar I. Perineural invasion in 
head and neck cancers - a review. J Med Life 2014;7:121-3.

10. El-Mofty SK. Histopathologic risk factors in oral and oropharyngeal squamous cell carcinoma variants: an update with special reference to HPV-related carcinomas. Med Oral Patol Oral Cir Bucal 2014;19:e377-85.

11. Dok R, Nuyts S. HPV Positive Head and Neck Cancers: Molecular Pathogenesis and Evolving Treatment Strategies. Cancers (Basel) 2016;8. pii: E41.

12. Yim EK, Park JS. The role of HPV E6 and E7 oncoproteins in HPV-associated cervical carcinogenesis. Cancer Res Treat 2005;37:319-24.

13. Bur AM, Lin A, Weinstein GS. Adjuvant radiotherapy for early head and neck squamous cell carcinoma with perineural invasion: A systematic review. Head Neck 2016;38 Suppl 1:E2350-7.

14. Miller ME, Palla B, Chen Q, et al. A novel classification system for perineural invasion in noncutaneous head and neck squamous cell carcinoma: histologic subcategories and patient outcomes. Am J Otolaryngol 2012;33:212-5.

15. Fagan JJ, Collins B, Barnes L, et al. Perineural invasion in squamous cell carcinoma of the head and neck. Arch Otolaryngol Head Neck Surg 1998;124:637-40.

16. Kurtz KA, Hoffman HT, Zimmerman MB, et al. Perineural and vascular invasion in oral cavity squamous

Cite this article as: da Silva SD, Kowalski LP. Perineural invasion in oral cancer: challenges, controversies and clinical impact. Chin Clin Oncol 2019;8(Suppl 1):S5. doi: 10.21037/ cco.2018.11.04 carcinoma: increased incidence on re-review of slides and by using immunohistochemical enhancement. Arch Pathol Lab Med 2005;129:354-9.

17. Yang X, Tian X, Wu K, et al. Prognostic impact of perineural invasion in early stage oral tongue squamous cell carcinoma: Results from a prospective randomized trial. Surg Oncol 2018;27:123-8.

18. Le Tourneau C, Jung GM, et al. Prognostic factors of survival in head and neck cancer patients treated with surgery and postoperative radiation therapy. Acta Otolaryngol 2008;128:706-12.

19. Tai SK, Li WY, Yang MH, et al. Treatment for T1-2 oral squamous cell carcinoma with or without perineural invasion: neck dissection and postoperative adjuvant therapy. Ann Surg Oncol 2012;19:1995-2002.

20. Pilch BZ, Gillies E, Houck JR, et al. Upper Aerodigestive tract Cancer Protocols and Checklist. Northfield, Ill: College of American Pathologists; 2005.

21. Cracchiolo JR, Xu B, Migliacci JC, et al. Patterns of recurrence in oral tongue cancer with perineural invasion. Head Neck 2018;40:1287-95.

22. National Comprehensive Cancer Network. Available online: https://www.nccn.org/about/news/ebulletin/ebulletindetail. aspx? ebulletinid=1340. Accessed September 2018. 